\section{Intravital high-resolution optical imaging of individual vessel response to photodynamic treatment}

\author{
Mamta Khurana, Eduardo H. Moriyama, \\ Adrian Mariampillai, and Brian C. Wilson* \\ University of Toronto, Ontario Cancer Institute, Division of \\ Biophysics and Bioimaging, Department of Medical \\ Biophysics, Toronto M5G2M9, Canada
}

\begin{abstract}
Intravital imaging using confocal microscopy facilitates high-resolution studies of cellular and molecular events in vivo. We use this, complemented by Doppler optical coherence tomography (OCT), to assess blood flow in a mouse dorsal skin-fold window chamber model to image the response of individual blood vessels to localized photodynamic therapy (PDT). Specific fluorescent cell markers were used to assess the effect on the vascular endothelial cell lining of the treated vessels. A fluorescently tagged antibody against an endothelial transmembrane glycoprotein (CD31) was used to image endothelial cell integrity in the targeted blood vessel. A cell permeability (viability) indicator, SYTOX Orange, was also used to further assess damage to endothelial cells. A fluorescently labeled anti-CD41 antibody that binds to platelets was used to confirm platelet aggregation in the treated vessel. These optical techniques enable dynamic assessment of responses to PDT in vivo, at both the vascular endothelial cell and whole vessel levels. $\odot 2008$ Society of Photo-Optical Instrumentation Engineers. [DOI: 10.1117/1.2965545]
\end{abstract}

Keywords: intravital; confocal microscopy; optical coherence tomography (OCT); photodynamic therapy (PDT); vascular; endothelial.

Paper 08129LR received Apr. 19, 2008; revised manuscript received May 16, 2008; accepted for publication May 29, 2008; published online Aug. 29, 2008.

Recent advances in optical imaging technologies, particularly confocal microscopy and optical coherence tomography (OCT), have facilitated real-time in vivo studies of cells and tissues in animal models, making possible assessment of anatomic, physiologic, metabolic, and pathologic information at the single-cell and molecular levels. ${ }^{1}$ Intravital microscopy has become pivotal in many fields, including developmental biology, ${ }^{2}$ immune system biology, ${ }^{3}$ neuropathology, and vascular biology. ${ }^{5}$ To date, in the field of vascular biology, the imaging of leukocyte rolling, ${ }^{6}$ thrombus formation, ${ }^{7}$ and molecular expression in endothelial cells ${ }^{8}$ have been reported.

Here, we used intravital confocal microscopy and Doppler OCT to examine the vascular response to localized photodynamic therapy (PDT). PDT, the combined action of a photosensitizer and light, is currently an approved therapy for several cancers and noncancerous conditions, including agerelated macular degeneration (AMD) ${ }^{9,10}$ in which permanent

*Tel: (416) 946-2952; E-mail: wilson@uhnres.utoronto.ca
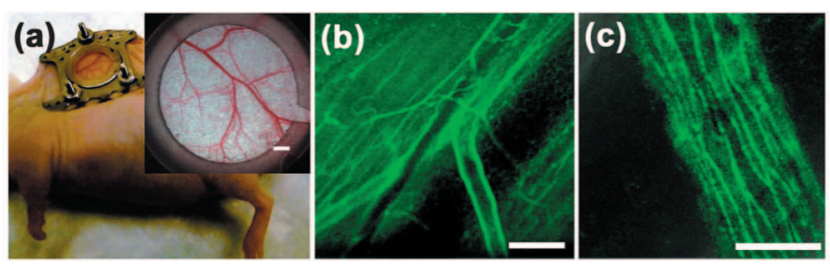

Fig. 1 Nude mouse (NCRNU-M) with transparent dorsal window and vascular pattern shown in insert (scale bar $1 \mathrm{~mm}$ ); Confocal images of endothelial cells labeled with FITC conjugated anti-CD31: (b) showing a $60 \pm 6 \mu \mathrm{m}$ diameter artery (scale bar $200 \mu \mathrm{m}$ ); (c) demonstrating individual endothelial cells lining a blood vessel (scale bar $50 \mu \mathrm{m}$ ).

closure of the abnormal choroidal neovascularization is the clinical goal, using the photosensitizer Visudyne (QLT, Inc., Vancouver, British Columbia, Canada). In vascular-targeted PDT, the endothelial cells lining the lumenal surface are believed to be the primary target, the killing of which instigates the vascular response. The damaged endothelium releases procoagulant as well as vasoactive factors, leading to vasoconstriction and/or blood flow stasis. ${ }^{11}$

We and our collaborators have recently reported successful closure of individual blood vessels in both the chorioallantoic membrane $(\mathrm{CAM})$ model $^{12}$ and the dorsal window chamber model $^{13}$ using two-photon PDT with highly-targeted focal light irradiation. Here, using Visudyne single-photon PDT in the window chamber model, ${ }^{13}$ we investigated in detail the vascular-PDT response in vivo at the single blood vessel and vascular endothelial cell levels. As shown in Fig. 1(a), in this model, a transparent window is surgically placed into the dorsal skin of nude mice to allow direct visualization and PDT treatment of the blood vessels. The thickness of the tissue in the chamber is $\sim 400 \mu \mathrm{m}$.

In order to examine the effects of treatment on the vascular endothelial cells, we injected a fluorescently labeled monoclonal antibody (Invitrogen, Burlington, Ontario, Canada) against platelet endothelial cell adhesion molecule-1 (PECAM-1, also referred as CD31) into the tail vein. This $\mathrm{mAb}$ reacts with $\mathrm{CD} 31$, a transmembrane glycoprotein of the immunoglobin superfamily that plays an important role in cell-cell adhesion, and is also involved in angiogenesis. ${ }^{14}$ It is expressed constitutively on endothelial cells and at lower density on platelets, neutrophils, monocytes, and a subset of $\mathrm{T}$ cells. ${ }^{14}$

Using excitation of the fluorophore fluorescein isothiocyanate (FITC) conjugated to anti-CD31 $(0.8 \mathrm{mg} / \mathrm{kg})$ with an argon-ion laser $(488 \mathrm{~nm}, 10 \times$ objective, NA 0.5$)$ coupled to a confocal microscope (LSM 510 Meta NLO; Carl Zeiss, Toronto, Ontario, Canada), we could obtain high-resolution images of the endothelial lining in vivo at the cellular level. The elongated cells shown in Fig. 1(c) are the individual endothelial cells lining a blood vessel [Fig. 1(b)].

To assess the PDT response, a small region (80 $\times 80 \mu \mathrm{m})$ of an artery, marked by the white box in Fig. 2(a), was irradiated by raster scanning with a $488 \mathrm{~nm}$ laser beam $(5 \times$ objective, spot size $1.2 \mu \mathrm{m}) 15 \mathrm{~min}$ after intravenous injection of $2.5 \mathrm{mg} / \mathrm{kg}$ body wt. of Visudyne. At this time, the photosensitizer is primarily in the vasculature. The red

1083-3668/2008/13(4)/040502/3/\$25.00 @ 2008 SPIE 


\section{JBO LETTERS}
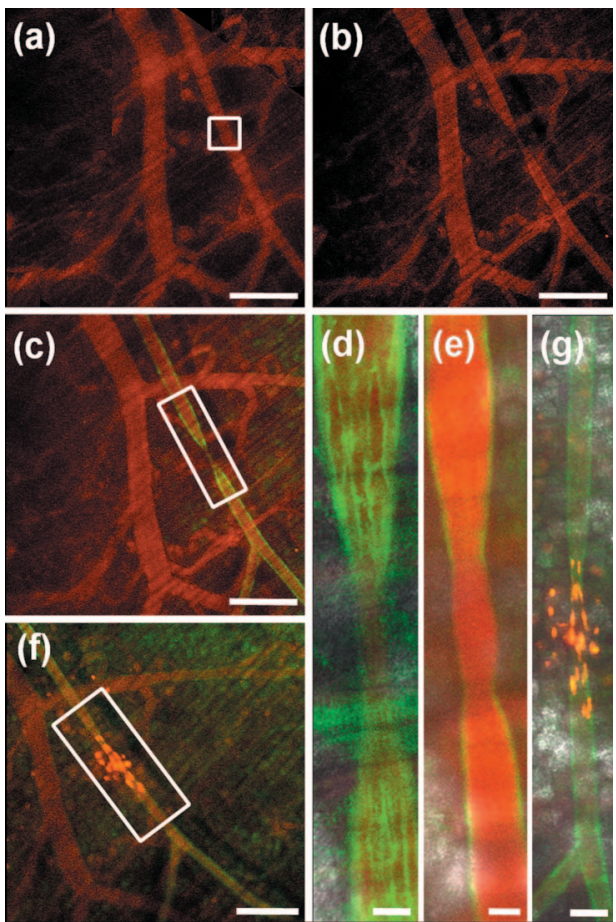

Fig. 2 Confocal microscope images of an artery-vein pair: (a) before and (b) immediately after PDT; (c) and (d) $1.5 \mathrm{~h}$, (e) $2.5 \mathrm{~h}$, and (f) and (g) $3 \mathrm{~h}$ after PDT. The white box over the $40 \pm 4 \mu \mathrm{m}$ ) diameter artery in (a) indicates the irradiated region. (d) and (g) are zoom images of the regions outlined in (c) and (f), respectively. The red fluorescence is from Visudyne. The green fluorescence in (c) to (g) is from the FITC-CD31 mAb conjugate. Orange-yellowish fluorescence in $(f)$ and (g) indicates dead cells labeled with SYTOX Orange. The scale bars are $200 \mu \mathrm{m}$ in (a), (b), (c), and (f), $20 \mu \mathrm{m}$ in (d) and (e), and $50 \mu \mathrm{m}$ in (g). Images are acquired using low power (light dose $1.3 \mathrm{~J} \mathrm{~cm}^{-2}$, incident intensity $101 \mathrm{~mW} \mathrm{~cm}^{-2}$, pixel dwell time $2.88 \mu \mathrm{s}$ ).

fluorescence of Visudyne was imaged during treatment using a $650-710 \mathrm{~nm}$ bandpass filter. A light dose of $451 \pm 43 \mathrm{~J} \mathrm{~cm}^{-2}$ (incident intensity $1340 \pm 124 \mathrm{~mW} \mathrm{~cm}^{-2}$, pixel dwell time $1.60 \mu \mathrm{s}$ ) was deposited in the marked region of a $40 \pm 4 \mu \mathrm{m}$ diameter artery, resulting in complete shrinkage in the targeted region [Fig. 2(b)] during the 6 min irradiation. (Video 1). The light dose at which the artery begins to constrict corresponds to $260 \mathrm{~J} \mathrm{~cm}^{-2}$.

In order to image the endothelial cell response in this region, $50 \mathrm{~min}$ following PDT, we injected the FITC-labeled

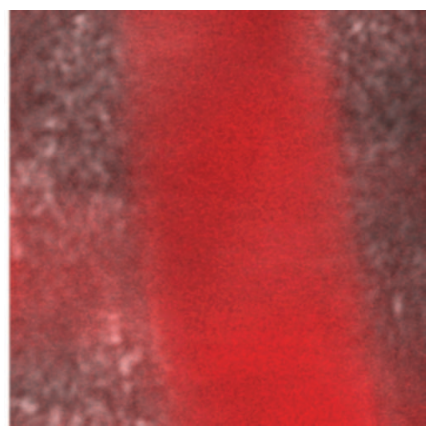

Video 1 PDT response of the targeted region. (QuickTime, $33 \mathrm{MB}$ ). [URL: http://dx.doi.org/10.1117/1.2965545.1].
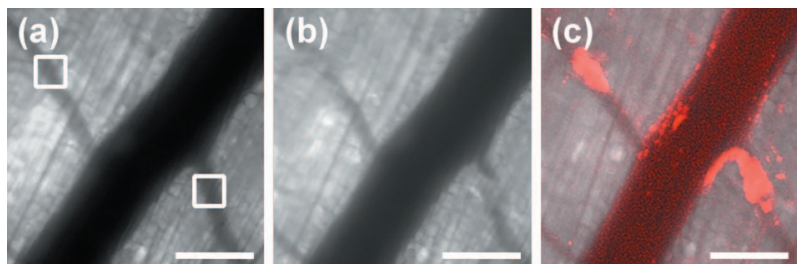

Fig. 3 Confocal microscope image (a) before, (b) immediately after, and (c) $1.5 \mathrm{~h}$ after PDT treatment. The white boxes $(80 \times 80 \mu \mathrm{m})$ indicate the two treated regions $\left(488 \mathrm{~nm}, 5 \times\right.$ objective, $124 \pm 12 \mathrm{~J} \mathrm{~cm}^{-2}$ $15 \mathrm{~min}$ after $16 \mathrm{mg} / \mathrm{kg}$ intravenous Visudyne injection). The antiCD41 antibody red fluorescence is due to phycoerythrin conjugated to the platelet marker. The scale bars are $200 \mu \mathrm{m}$.

antibody intravenously $(0.8 \mathrm{mg} / \mathrm{kg})$. As seen in Figs. 2(c) and 2(d), this showed specific binding to the endothelial cells on either side of the treated region, but absence of binding in the irradiated region. (These images were acquired using the $488 \mathrm{~nm}$ laser set to 5 times lower power so that there was minimal additional PDT effect.) Following this, a cell permeability indicator, SYTOX Orange, was injected intravenously $(2 \mathrm{mM} / \mathrm{kg})$. This enters cells with compromised plasma membrane but is excluded from live cells. ${ }^{15}$ Selective staining can be seen in the treated region [Figs. 2(f) and 2(g)], indicating endothelial cell damage. Figure $2(\mathrm{~g})$ also shows that the endothelial cell lining of the artery is no longer intact. This confirms our previous in vitro results ${ }^{16}$ and other reports ${ }^{10}$ of endothelial cell rounding and disruption of cell monolayers following PDT. In addition, Chen et al. showed that the endothelial intracellular gaps formed after PDT are due to microtubule depolarization. ${ }^{10}$

Subsequent to endothelial denudation, platelet adherence to the subendothelium and exposed media occurs. ${ }^{17}$ In order to confirm this in vivo, we injected a fluorescently labeled anti-CD41 antibody (BD Biosciences, Mississauga, Ontario, Canada) that reacts with glycoprotein (gp) IIb (also called integrin $\alpha \mathrm{II}$ chain) and associates with the integrin $\beta 3$ chain (gpIIIa or CD61) to form the gpIIb/IIIa (CD41/CD61) complex. CD41/CD61 is expressed on platelets, megakaryocytes, and early hematopoietic progenitors. The integrin complex binds to fibrinogen, fibronectin, vitronectin, von Willebrand factor, and thrombospondin. Figure 3(a) shows two $40 \pm 4 \mu \mathrm{m}$ diameter veins that were locally treated with PDT. Figure 3(b) shows the treated regions immediately after treatment. A phycoerythrin labeled CD41 antibody was injected 50 min following PDT, and Fig. 3(c) shows platelet aggregation and shedding selectively in the treated regions. The thrombus in the irradiated veins was monitored in real time for $1 \mathrm{~h}$ (see Video 2).
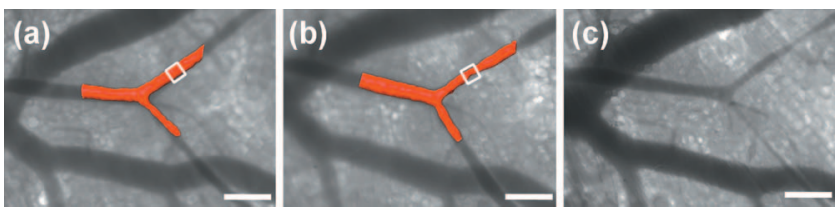

Fig. 4 3-D-rendered images of blood flow (red) produced by Doppler OCT imaging (a) before, (b) $2 \mathrm{~h}$ after, and (c) $16 \mathrm{~h}$ after treatment, overlaid on the confocal microscope images. The white box indicates the irradiated region. The scale bars are $200 \mu \mathrm{m}$. 


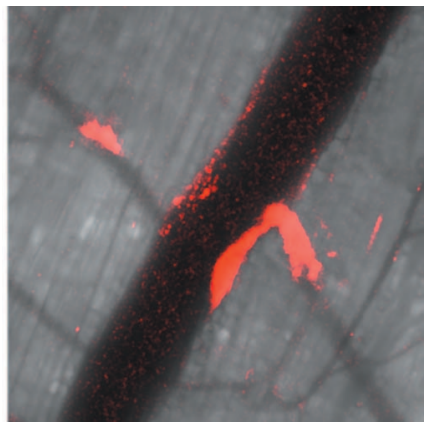

Video 2 Thrombus in the irradiated vein monitored in real time for $1 \mathrm{~h}$ (QuickTime, 386 MB).

[URL: http://dx.doi.org/10.1117/1.2965545.2].

We also used Doppler OCT (DOCT) to record blood flow pre- and post-irradiation in the targeted and nearby vessels. For this, a 24-kHz swept laser source scanning 1260 to $1360 \mathrm{~nm}$ was used to map the tissue structure with a spatial resolution of $10 \mu \mathrm{m}$, and the Doppler frequency shift induced by moving red blood cells was used to measure the flow velocity. ${ }^{18}$ Figures 4(a)-4(c) show, respectively, pre-, $2 \mathrm{~h}$ post-, and $16 \mathrm{~h}$ post-irradiation images. The blood flow recorded by DOCT (shown in red) is overlaid on the respective confocal microscope images. With a $75^{\circ}$ Doppler angle, the peak velocity pre-PDT in the targeted artery was $812 \pm 122 \mu \mathrm{m} \mathrm{s}^{-1}$, changing to $3282 \pm 492,1539 \pm 231$, and $0 \mu \mathrm{m} \mathrm{s}^{-1}$ immediately after, $2 \mathrm{~h}$ later, and $16 \mathrm{~h}$ postirradiation, respectively. The marked increase in blood flow velocity immediately after PDT could be due to narrowing of the lumen following vasoconstriction. Two hours later, the blood flow decreased but was still faster than pre-PDT. This could be due to platelet aggregation in the treated region. No blood flow was detected $16 \mathrm{~h}$ later in the treated artery or in the feeding artery.

In summary, we have demonstrated the feasibility and utility of intravital confocal microscopy combined with DOCT to image single-vessel responses to PDT. Previous in vivo vascular-targeted $\mathrm{PDT}^{11}$ generally irradiated a large region of tissue, comprising multiple arteries and veins. Here, we localized the damage selectively in a single targeted vessel. This can also be done using two-photon PDT. ${ }^{13}$ Using in vivo imaging tools; we showed that the localized-PDT response is similar to that reported in previous studies when large areas were irradiated. We plan now to use these techniques to examine the photosensitizer and light dose responses of single blood vessels to localized PDT and, in particular, how this depends on the vessel type and diameter and the irradiation geometry. The goal is to provide information to help optimize two-photon PDT of AMD, including targeting of feeder vessels. ${ }^{19}$ With the two-photon illumination, excitation in the $z$ direction is limited to $\sim 20 \mu \mathrm{m}$, whereas it extends throughout the entire $z$ length in case of one-photon illumination.

Similar approaches could be used to investigate and understand the effects of other treatments such as focal photothermal or photochemical treatments.

\section{Acknowledgments}

This work was supported by the Canadian Institute for Photonic Innovations. E. H. Moriyama was supported in part by
National Institutes of Health Grant No. CA43892, and A. Mariampillai by Canadian Institutes of Health Research Grant No. 82498. The authors also thank QLT, Inc. (Vancouver, British Columbia, Canada), for providing Visudyne. James Jonkman and Miria Bartolini of the Advanced Optical Microscopy Facility, UHN, provided technical assistance.

\section{References}

1. S. R. Cherry, "In vivo molecular and genomic imaging: new challenges for imaging physics," Phys. Med. Biol. 49, R13-48 (2004).

2. P. Dahm-Kahler, C. Lofman, R. Fujii, M. Axelsson, P. O. Janson, and M. Brannstrom, "An intravital microscopy method permitting continuous long-term observations of ovulation in vivo in the rabbit," Hum. Reprod. 21, 624-631 (2006).

3. R. N. Germain, M. J. Miller, M. L. Dustin, and M. C. Nussenzweig, "Dynamic imaging of the immune system: progress, pitfalls, and promise," Nat. Rev. Immun. 6, 497-507 (2006).

4. T. Misgeld and M. Kerschensteiner, "In vivo imaging of the diseased nervous system," Nat. Rev. Neurosci. 7, 449-463 (2006).

5. B. Furie and B. C. Furie, "In vivo thrombus formation," J. Thromb. Haemost. 5, 12-17 (2007).

6. L. Sikora, A. C. Johansson, S. P. Rao, G. K. Hughes, D. H. Broide, and P. Sriramarao, "A murine model to study leukocyte rolling and intravascular trafficking in lung microvessels," Am. J. Pathol. 162, 2019-2028 (2003).

7. S. Falati, P. Gross, G. Merrill-Skoloff, B. C. Furie, and B. Furie, "Real-time in vivo imaging of platelets, tissue factor, and fibrin during arterial thrombus formation in the mouse," Nat. Med. 8, 11751181 (2002).

8. J. M. Runnels, P. Zamiri, J. A. Spencer, I. Veilleux, X. Wei, A. Bogdanov, and C. P. Lin, "Imaging molecular expression on vascular endothelial cells by in vivo immunofluorescence microscopy," Mol. Imaging 5, 31-40 (2006).

9. M. A. Awan and S. A. Tarin, "Review of photodynamic therapy," Surgeon. 4, 231-236 (2006).

10. B. Chen, B. W. Pogue, P. J. Hoopes, and T. Hasan, "Vascular and cellular targeting for photodynamic therapy," Crit. Rev. Eukaryot Gene Expr 16, 279-306 (2006).

11. V. H. Fingar, "Vascular effects of photodynamic therapy," J. Clin. Laser. Surg. Med. 14, 323-328 (1996).

12. K. S. Samkoe, A. A. Clancy, A. Karotki, B. C. Wilson, and D. T. Cramb, "Complete blood vessel occlusion in the chick chorioallantoic membrane using two-photon excitation photodynamic therapy: implications for treatment of wet age-related macular degeneration," J. Biomed. Opt. 12, 034025 (2007).

13. H. A. Collins, M. Khurana, E. H. Moriyama, A. Mariampillai, E. Dahlstedt, M. Balaz, M. K. Kuimova, M. Drobizhev, V. X. D. Yang, D. Phillips, A. Rebane, B. C. Wilson, and H. L. Anderson, "Blood vessel closure using photosensitizers engineered for two-photon excitation," Nat. Photonics 2, 420-424, 2008).

14. H. M. DeLisser, M. Christofidou-Solomidou, R. M. Strieter, M. D. Burdick, C. S. Robinson, R. S. Wexler, J. S. Kerr, C. Garlanda, J. R. Merwin, J. A. Madri, and S. M. Albelda, "Involvement of endothelial PECAM-1/CD31 in angiogenesis," Am. J. Pathol. 151, 671-677 (1997).

15. M. E. Dailey and M. Waite, "Confocal imaging of microglial cell dynamics in hippocampal slice cultures," Methods 18, 222-230 (1999).

16. A. Karotki, M. Khurana, J. R. Lepock, and B. C. Wilson, "Simultaneous two-photon excitation of Photofrin in relation to photodynamic therapy," Photomed. Photobiol. 82, 443-452 (2006).

17. P. Fungaloi, R. Statius van Eps, Y. P. Wu, J. D. Blankensteijn, P. de Groot, H. van Urk, R. van Hillegersberg, and G. LaMuraglia, "Platelet adhesion to photodynamic therapy-treated extracellular matrix proteins," Photomed. Photobiol. 75, 412-417 (2002).

18. A. Mariamplillai, B. Standish, C. Randall, G. Liu, N. R. Munce, I. A. Vitkin, A. Cable, J. Jiang, and V. X. D. Yang, "Optical cardiogram gated 2-D doppler flow imaging at $1000 \mathrm{fps}$ and 4-D imaging at $36 \mathrm{fps}$ on a swept source OCT system," Opt. Express 15, 1627-1638 (2007).

19. R. W. Flower, "Optimizing treatment of choroidal neovascularization feeder vessels associated with age-related macular degeneration," Am. J. Ophthalmol. 134, 228-239 (2002). 